\title{
EDRL-MDL: Metaheurística para el enrutamiento y asignación de longitud de onda en tráfico dinámico
}

\author{
DLE-WDM:Metaheuristic to routing and wavelength \\ assignment in dynamic traffic \\ Arturo Rodríguez García $^{1 *} \quad$ Juan Carlos Travieso ${ }^{1} \quad$ Fideromo Saavedra Guzmán $^{2}$ \\ Leonardo Ramírez López ${ }^{3}$ \\ Recibido 9 de diciembre de 2015, aceptado 12 de octubre de 2016 \\ Received: December 9, 2015 Accepted: October 12, 2016
}

\begin{abstract}
RESUMEN
Las heurísticas utilizadas para la solución del problema de enrutamiento y asignación de longitud de onda en redes ópticas en escenarios dinámicos han tenido resultados parcialmente buenos y sobre todo no responden bien cuando son sometidas a estrés. El presente artículo se propone una nueva estrategia llamada Snake-Two que utiliza el algoritmo Snake-One junto al monitoreo de los enlaces de la red, procurando concentrar el tráfico en los sectores más utilizados dejando más disponibilidad en el resto de la red, esto permite que disminuya la probabilidad de bloqueo instantánea de las solicitudes entrantes a la red. Los resultados obtenidos mejoran la probabilidad de bloqueo media hasta en $37,7 \%$ de los resultados más altos obtenidos; sin embargo, la utilización de la red continúa su aumento. Estos resultados permiten garantizar que la atención de solicitudes mejora para escenarios inferiores a los 140 Erlangs.
\end{abstract}

Palabras clave: Snake-One, algoritmos heurísticos, NSFNET, Snake-Two.

\begin{abstract}
The heuristics used to solve the problem of routing and wavelength assignment in optical networks in dynamic scenarios had been partially successful and especially do not respond well when subjected to estrés. This article describes a new strategy called Snake-Two that uses the algorithm Snake-One with the monitoring of network links, trying to concentrate on the most used traffic areas leaving more openings in the rest of the network is proposed, which allows decreasinge the likelihood of blocking incoming network requests. The results improve the average probability of blocking up to $37.7 \%$ of the highest obtained results. However, the use of the network continues to increase. These results ensure that the improved care of requests for lower scenarios to 140 Erlangs.
\end{abstract}

Keywords: Snake-One, heuristic algorithm, NSFNET, Snake-Two.

\footnotetext{
1 Departamento de Tecnologías Industriales. Facultad Tecnológica. Universidad de Santiago de Chile. Avenida Ecuador 3659. Estación Central. Santiago, Chile. E-mail: arturo.rodriguez@usach.cl; juancarlos.travieso@usach.cl

2 Departamento de Ingeniería Eléctrica. Facultad de Ingeniería. Universidad de Santiago de Chile. Avenida Ecuador 3659. Estación Central. Santiago, Chile. E-mail: fideromo.saavedra@usach.cl

3 Departamento de Ingeniería Eléctrica. Universidad Militar de Nueva Granada. Bogotá, Colombia. E-mail: leonardo.ramirez@ unimilitar.edu.co

* Autor de correspondencia.
} 


\section{INTRODUCCIÓN}

Las redes ópticas WDM (Wavelength Division Multiplexing) o Multiplexación por división de Longitud de Onda (MDL) sin conversión de longitud de onda son actualmente las redes más estudiadas y la problemática radica en buscar la ruta entre el nodo origen y el nodo destino y la longitud de onda asociada que además tiene la restricción de ser la misma a lo largo de toda la ruta. Estas redes están sometidas a dos tipos de tráficos conocidos como estático y dinámico. El escenario estático permite la búsqueda de soluciones óptimas, debido a que el universo disponible se mantiene constante por largos períodos; mientras que el escenario dinámico no permite optimización debido a que el universo disponible cambia constantemente, por lo que solo se buscan buenas rutas. Los algoritmos heurísticos son procesos que permiten encontrar soluciones rápidas, pero no necesariamente óptimas. Se han realizado estudios con algoritmos heurísticos como genéticos, Simulated Annealing, búsqueda tabú y Snake-One comparándolos mediante indicadores como probabilidad de bloqueo $\left(P_{B}\right)$ y utilización de la red $\left(U_{R}\right)$ [11-12]; los que no han mejorado sustancialmente. Hoy se desarrollan diferentes meta heurísticas que procuran mejorar los resultados de estas heurísticas $[1-4,9,11]$.

La metaheurística mostrada en este artículo se denomina Snake-Two, que utiliza el algoritmo SnakeOne pero concentrando el tráfico en los enlaces más utilizados (LMU - Link More Used) buscando saturar la operación del enlace (máxima capacidad); de esta forma los enlaces menos utilizados estarán menos demandados por los lightpath y podrán atender la demanda entrante, y de esta manera disminuir la $P_{B}$ de la red $[1,6,8]$.

\section{ROUTING AND WAVELENGTH ASSIGNMENT}

La capacidad de servicio de las redes ópticas depende de la cantidad de longitudes de onda que utilizan y del tipo de operación como con reutilización de longitud de onda (WR-Wavelength Reusing) o con continuidad de longitud de onda (WC-Wavelength Continuity) (ver ecuaciones (1) y (2), respectivamente). Los lightpath o caminos de luz están formados por rutas y longitudes de onda asociadas a cada enlace.

$$
L P_{08}=\left(e_{01}, \lambda_{0}\right)-\left(e_{13}, \lambda_{1}\right)-\left(e_{38}, \lambda_{3}\right)
$$

En la ecuación (1), observamos un lipghtpat que va del nodo 0 al nodo 8 pasando por tres enlaces y utiliza una longitud de onda diferente en cada enlace, este tipo de lightpaths son posibles en redes WDM-WR y es conocido como reutilización de longitud de onda. Las redes sujetas a la restricción de continuidad de longitud de onda (WDM-WC), solo pueden obtener lighpaths como se observan en la ecuación (2).

$$
L P_{08}=\left(e_{01}, \lambda\right)-\left(e_{13}, \lambda\right)-\left(e_{38}, \lambda\right)
$$

Los actuales escenarios de redes WDM son con WC y son utilizadas para redes ópticas DWDM (Dense Wavelength Division Multiplexing) utilizada para grandes distancias con un máximo de 160 longitudes de onda, por lo que no es necesario reutilizar la longitud de onda (WR); sin embargo, si la demanda aumenta y no existe posibilidad de un aumento de longitudes de onda, entonces será necesario reutiliza la longitud de onda. Las redes DWDM al multiplexar mayor cantidad de longitudes de onda tienen un espacio entre canal de 50 a $200 \mathrm{Ghz}(0,2-1,6 \mathrm{~nm})$ [7].

Estas redes al trabajar con más longitudes de onda en un rango donde la atenuación es baja (Coeficiente de atenuación bajo - Tercera Ventana) tienen la ventaja de no necesitar WR, pero la desventaja de la aparición de fenómenos no lineales como FWM (Four Wave Mixing - Mezcla de Cuatro Ondas) entren otros, causando problemas en la recepción de la señal óptica $[1,4]$.

El problema RWA o enrutamiento y asignación de longitud de onda, se plantea como la búsqueda de una ruta y una longitud de onda asociada que satisfaga la demanda solicitada, como se observa en la ecuación (3).

$$
S_{i}^{h}=\left(n_{i}, n_{D}, n_{C X}, t_{C X}\right)
$$

Donde, $S_{i}^{h}$ representa la $h$-ésima solicitud entrante al $i$-ésimo nodo, $n_{i}$ representa el nodo origen, $n_{D}$ representa el nodo destino, $n_{C X}$ representa el número de conexiones solicitadas y $n_{C X}$ representa el tiempo de conexión solicitado o Holding Time en milisegundos [8, 10, 13-14]. 


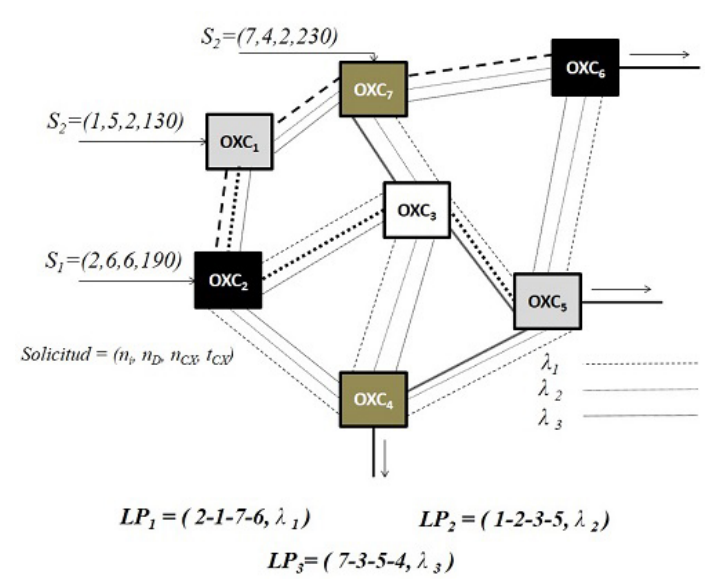

Figura 1. Problema RWA.

La solución del RWA para el escenario estático es conocida como SLE (Static LightPath Establishment) o Establecimiento Estático de Rutas de Luz (EERL), tal como se dijo anteriormente este escenario es optimizable, donde se utilizan algoritmos convencionales. El escenario dinámico es conocido como DLE (Dynamic LightPath Establishment) o establecimiento dinámico de rutas de luz (EDRL). El DLE utiliza algoritmos heurísticos por la rapidez de la solución, por la cantidad de soluciones y su simplicidad operativa $[1,4,15]$.

\section{SNAKE TWO ALGORITHM}

Los resultados mostrados en [1] mostraron que la $P_{B}$ disminuye mientras que la $U_{R}$ aumentaba, la metaheuristica Snake-Two se plantea con la finalidad de disminuir la probabilidad de bloqueo media de la red a un bajo incremento de la utilización de la red.

La metaheurística procura concentrar el tráfico de la red basado en un principio llamado "Enlace más utilizado" (LMU - Link More Used). Los lightpaths deberán privilegiar su tránsito por los enlaces que son más utilizados hasta el límite de saturación o congestión. De esta forma los enlaces menos utilizados se verán disponibles para la demanda entrante o futura, disminuyendo la probabilidad de bloqueo instantánea.

Para lograr este principio se monitoriza el tránsito de los enlaces ordenándolos del más transitado al menos transitado (de mayor a menor costo). Para ello se crea la matriz de Enlaces Congestionados (MCL - Matrix Congested Links), esta matriz contiene los costos instantáneos de la utilización de cada enlace de la red.

Para garantizar el paso por un enlace congestionado, cualquier solicitud deberá contener un enlaces de la matriz MCL.

La ecuación (4), representa la matriz MCL que guardara las características del tráfico actual en la red.

$$
M L C\left\{\begin{array}{l}
m_{i j k} / m_{i j k}=C I E_{i-j}, i \in[0, N(N=1)] \\
\wedge j=[0,2] \wedge k \in\left[0, n_{w}-1\right]
\end{array}\right\}
$$

Donde:

$m_{i j k} \quad$ : Elemento de la matriz de enlaces congestionados $(i, j, k)$

$C I E_{i-j}$ : Costo instantáneo del enlace $i-j$

$N \quad$ : Número de nodos de la red

$n_{x} \quad$ : Número de longitudes de onda por enlace en la red

Snake-One y sus procesos se pueden observar [1]. En la Figura 2 se observa la metaheurística Snake Two y el uso de Snake-One.

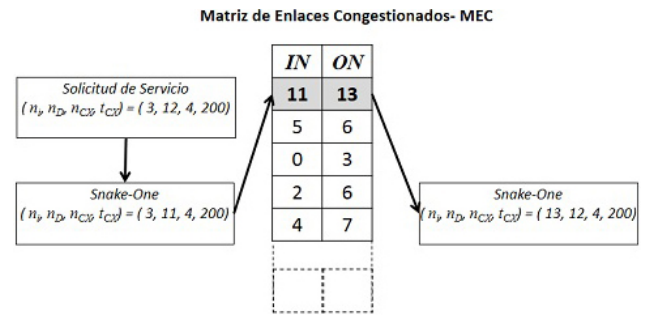

Figura 2. Snake-Two, mostrando la matriz MCL.

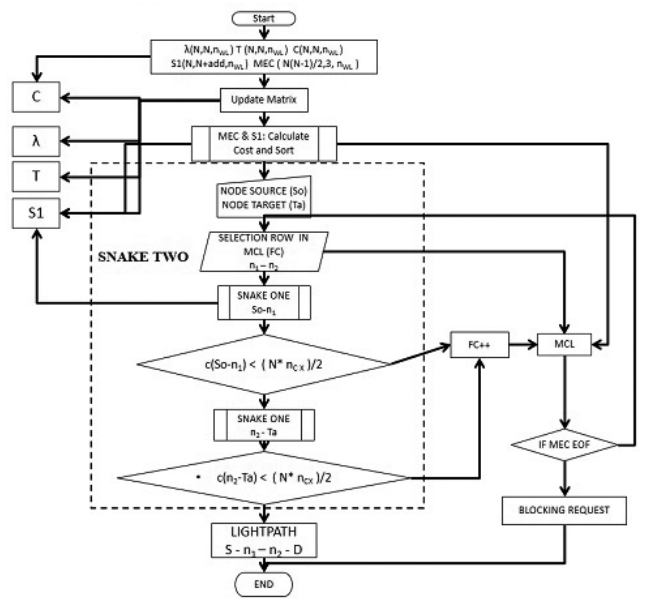

Figura 3. DFD Snake-Two. 
En la Figura 3, se presenta el diagrama de flujo del algoritmo, donde se observa que el algoritmo utiliza como paso previo un enlace de la tabla ordenada de enlaces congestionado de tal forma que obliga a la ruta a pasar por el enlace congestionado que más se ajuste a la solicitud, de esta forma se evita que la carga se distribuya por toda los enlaces de la red, controlando la disponibilidad de los enlaces menos congestionados, de esta forma siempre se podrá tener espacio para nuevas solicitudes, ayudando a la disminución de la probabilidad de bloqueo.

\section{ESCENARIO DE COMPARACIÓN}

La simulación se llevó a cabo bajo las mismas condiciones que en $[1,8,10,13,15]$.

La red utilizada fue la NSFNET (National Science Foundation NETwork) con 14 nodos, 21 enlaces de fibra óptica y 8 longitudes de onda, ver Figura 4 [5]. Se varió la carga en el intervalo $[0,180]$ con incrementos de 10 Erlangs, registrándose la probabilidad de bloqueo instantánea y la utilización de la red. El número total de conexiones realizadas durante la simulación fue de $10^{8}$ solicitudes de conexión.

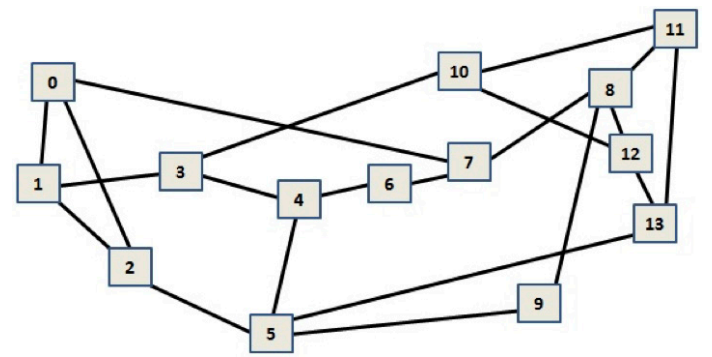

Figura 4. Red NSFENET utilizada.

\section{COMPARACIÓN DE RESULTADOS}

Los algoritmos heurísticos comparados son Snake One [1] y Snake Two utilizando como elementos referenciales algoritmos genéticos, simulated annealing y tabú search [8-10, 13].

Tabla 1. Comparación de valores medios.

\begin{tabular}{|c|c|c|c|c|c|}
\cline { 2 - 6 } \multicolumn{1}{c|}{} & \multicolumn{1}{c|}{ AG } & \multicolumn{1}{c|}{ SA } & \multicolumn{1}{c|}{ TS } & SNK1 & SNK2 \\
\hline$P_{B}$ & 0,42 & 0,45 & 0,36 & 0,32 & 0,28 \\
\hline$U_{R}$ & 42,17 & 45,30 & 55,17 & 68,2 & 72,2 \\
\hline
\end{tabular}

En la Tabla 1 se puede observar la comparación de los valores medios de los indicadores $P_{B}$ y $U_{R}$ para cada algoritmo heurístico.

Los valores medios muestran una disminución de la probabilidad de bloqueo pero a costa de un incremento del uso de los recursos de la red.

Con la finalidad de afinar la comparación de las heurísticas se incorporó otro indicador denominado "Ganancia Algorítmica - GAA" (Algorithmic GainALG) que relaciona el valor medio de la $U_{R}$ y el valor medio de la $P_{B}$, ver ecuación (2).

$$
G A A=\frac{U_{R}}{P_{B}}
$$

Donde:

$U_{R}$ : Utilización de la red media.

$P_{B}$ : Probabilidad de bloqueo media.

La ganancia algorítmica al relacionar la utilización de la red con la probabilidad de bloqueo media, indica cuanto recurso de red se utiliza por cada unidad de probabilidad de bloqueo media entregada, es decir, un algoritmo será más eficiente en la medida que tenga un bajo GAA.

Tabla 2. Comparación de la tasa de probabilidad de bloqueo media.

\begin{tabular}{|c|c|c|c|c|c|}
\cline { 2 - 6 } \multicolumn{1}{c|}{} & AG & SA & TS & SNK1 & SNK2 \\
\hline GAA & 100,05 & 100,24 & 154,38 & 215,90 & 255,38 \\
\hline
\end{tabular}

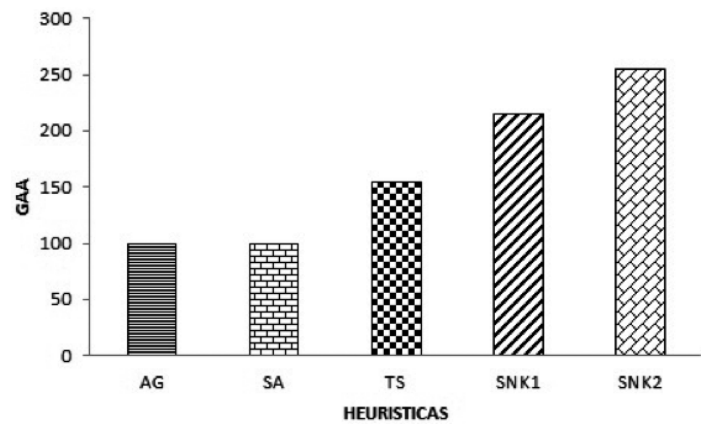

Figura 5. Comparación de la TAH para cada algoritmo heurístico.

Según la Tabla 2 y la Figura 5, muestran que la GAA que entrega mejores resultados promedio 
es el algoritmo genético, es decir, cuesta menos uso de la red entregar la misma probabilidad de bloqueo media, y además se observa que el SNK2 es el que más recursos de red consume; esto es un indicador que los lighpath de AG son más cortos que los de SNK2, es decir el número de saltos es menor en AG. En el caso de SNK2 al forzar su paso por enlaces de alta congestión se generan rutas más largas.

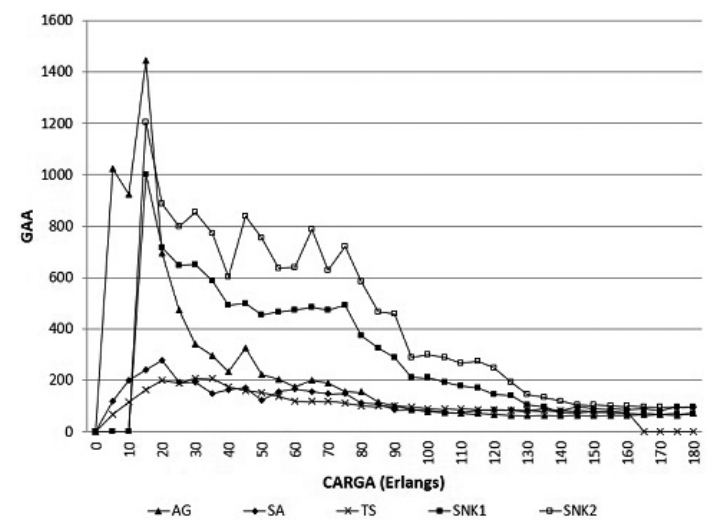

Figura 6. Distribución de la ganancia algorítmica para cada heurística en cada carga.

Sin embargo, en la Figura 6 se puede observar que la GAA del algoritmo genético es mejor para escenarios de bajo tráfico (140 Erlangs) y cuando trabajan con estrés los algoritmos tienen una GAA muy similar.

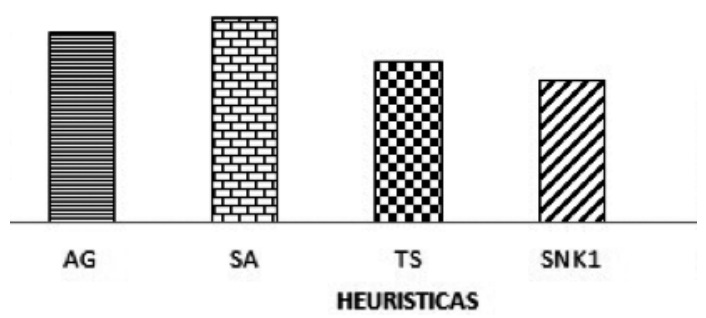

Figura 7. Comparación de la probabilidad de bloqueo media para cada algoritmo heurístico.

Observando la Figura 7, observamos que la probabilidad de bloqueo media de SNK2 mejora a SNK1 y es el menor de todas las heurísticas, sin duda, la estrategia funciona pero su debilidad está en que genera rutas largas y, por tanto, tiene una elevada utilización de la red como se vio anteriormente.

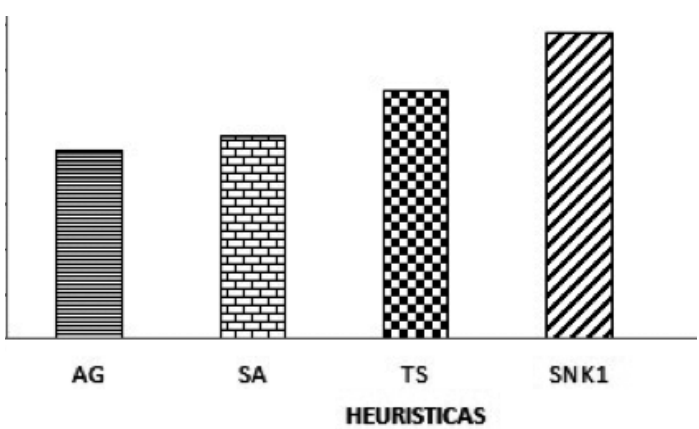

Figura 8. Comparación de la utilización de la red media para cada algoritmo heurístico.

En la Figura 8 podemos corroborar el aumento en el uso de los recursos por SNK2, al tener un valor medio más alto que todas las heurísticas en comparación.

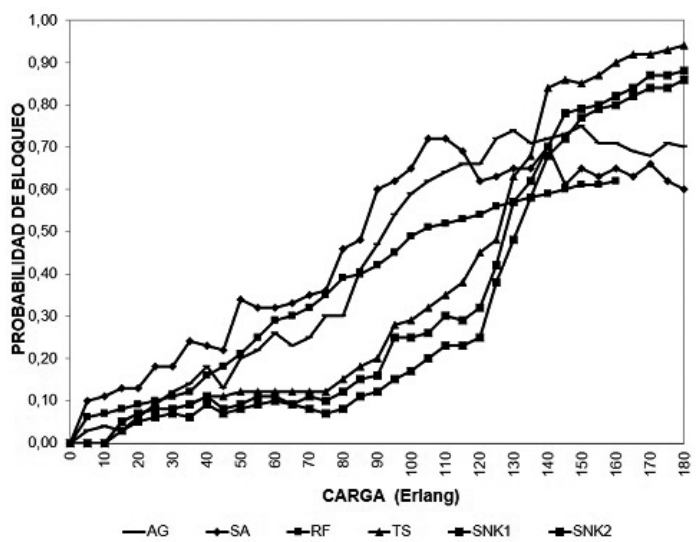

Figura 9. Probabilidad de bloqueo instantánea.

En la Figura 9 se observa el comportamiento muy bajo de la probabilidad de bloqueo instantánea para SNK2, pero se mantiene la característica de bajo PB del AG y SA sobre los 150 Erlangs. Estas últimas heurísticas tienen un robusto comportamiento para escenarios de estrés.

En la Figura 10 se muestra el aumento del uso de los recursos por SNK2 esto es, sin duda, una 


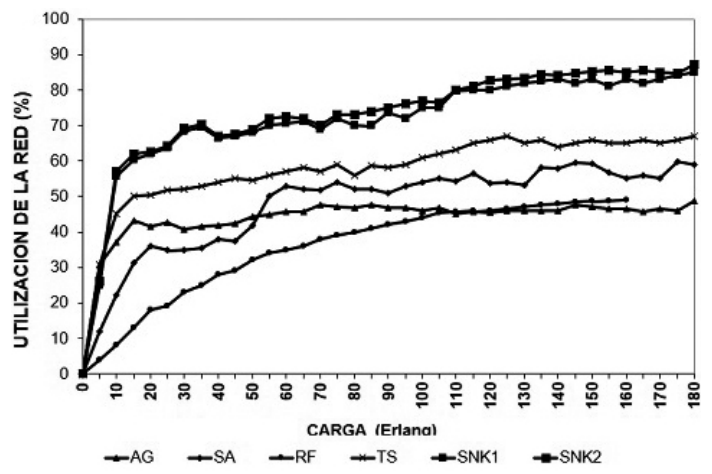

Figura 10. Utilización de la red.

característica de la metaheurística. SNK2 logra disminuir la probabilidad de bloqueo instantánea pero con un aumento del uso de los recursos de la red.

\section{CONCLUSIONES}

Snake Two muestra una mejora de la probabilidad de bloqueo media (disminución) pero por medio de un incremento del indicador utilización de la red, esto se debe a que SNK2 desarrolla rutas más largas. La GAA muestra claramente que el AG es el más eficiente de todas las heurísticas, pero para bajos tráficos TS, SNK1 y SNK2 tienen un mejor desempeño en la atención de las solicitudes de servicio (bajo $P_{B}$ ). Sin embargo, para escenarios de estrés AG y SA tienen una robustez que las otras heurísticas no logran igualar. Por lo demás, la estrategia de utilizar el LMU (Link More Used) junto al algoritmo SNK1 (Snake One) logra una disminución de la probabilidad de bloqueo y este efecto es importante porque ayuda a garantizar una atención oportuna de la demanda entrante; sin embargo, el aumento del uso de los recursos de red es un elemento a considerar sobre todo para escenarios de estrés, superiores a los 150 Erlangs.

\section{AGRADECIMIENTOS}

Se realiza un especial reconocimiento al programa DICYT de la Universidad de Santiago de Chile USACH (Proyecto DICYT No 061572RG), y al Proyecto Basal USA1555 por el importante apoyo a la investigación y en particular al Grupo de Investigación en Nuevas Tecnologías (GINT-DTI-USACH).

\section{REFERENCIAS}

[1] A. Rodríguez, J-C. Torres and L. Ramírez. "New heuristic algorithm for dynamic traffic in WDM optical networks". Ing. Investig. Vol. $35 \mathrm{~N}^{\circ}$ 3. 2015.

[2] S. Barat, A. Pradhan and T. De. "A genetic algorithm for multicasting in resource constraint WDM mesh networks". In Industrial and Information Systems (ICIIS), 20127 th IEEE International Conference on, pp. 1-6, 6-9. August, 2012. DOI: 10.1109/ ICIInfS.2012.6304820

[3] D. Monoyios and K. Vlachos. "Multiobjective Genetic Algorithms for Solving the Impairment-Aware Routing and Wavelength Assignment Problem". In Optical Communications and Networking, IEEE/ OSA Journal of. Vol. $3 \mathrm{~N}^{\circ}$ 1, pp. 40-47. January, 2011.

[4] I. Ruge, H. Numpaque, Y. Medina e I. Ortiz. "Estudio, caracterización y optimización del diseño de redes DWDM". Tecnura. Vol. 16 $\mathrm{N}^{\circ} 34$, pp. 43-55. 2012.

[5] G. Puerto, M. Sastoque y C. Suárez. "Dimensionado de multicast óptico para redes WDM de acceso dinámicas convergentes". Ingeniare. Rev. chil. ing. Vol. $23 \mathrm{~N}^{\circ} 2$, pp. 175-181. 2015.

[6] A. Rodríguez, L. Ramírez, L. Rivera and A. Gutiérrez. "Comparing Genetic Algorithms and Simulated Annealing for dynamic traffic routing". LNEE Springer. Vol. 315, pp. 3-14. 2014.

[7] Y. Pan, D. Hui and B. Ramamurthy. "Costoptimized joint resource allocation in grids/ clouds with multilayer optical network architecture". Optical Communications and Networking, IEEE/OSA Journal of. Vol. 6 $\mathrm{N}^{\circ}$ 10, pp. 911-924. 2014.

[8] A. Rodríguez, A. Gutiérrez, L. Rivera y L. Ramírez. "Ruteo y Asignación de Longitud de Onda: Comparación de Algoritmos Genéticos y Simulated Annealing”. Inf. Tecnol. Vol. 25 $\mathrm{N}^{\circ}$ 4, pp. 13-18. 2014.

[9] A. Rubio-Largo, M. Vega-Rodríguez, J. Gómez-Pulido y J. Sánchez-Pérez. "Multiobjective Metaheuristics for Traffic Grooming in Optical Networks". Evolutionary Computation, IEEE Transactions on, Vol. 17 $\mathrm{N}^{\circ}$ 4, pp. 457-473, 2013. 
[10] A. Rodríguez, L. Ramírez, L. Rivera y A. Gutiérrez. "Routing wavelength assignement: A solution with tabu search in dynamic traffic". Ingeniare. Rev. chil. ing. Vol. 22 No 4, pp. 495-503. 2014.

[11] S. Sakamoto, T. Oda, E. Kulla, M. Ikeda, L. Barolli and F. Xhafa. "Performance analysis of WMNs using simulated annealing algorithm for different temperature values". Complex, Intelligent, and Software Intensive Systems (CISIS). Taichung, Taiwan. Vol. 1, pp. 164-168. 2013.

[12] L. Khelifi, I. Zidi, K. Zidi and K. Ghedira. "A hybrid alpproach based on multi-objective simulated annealing and tabu search to solve the dynamic dial a ride problem". Advanced Logistics and Transport (ICALT). Sousse, Tunez. Vol. 1, pp. 227-232. 2013.

[13] A. Rodríguez y F. Saavedra. "Optimización del Algoritmo Genético para la Solución
Integral de Enrutamiento en Redes Fotónicas". Inf. Tecnol. Vol. $21 \mathrm{~N}^{\mathrm{o}} 3$, pp. 125-133. 2010.

[14] R. Barpanda, B. Sahoo, A. Turuk and B. Majhi. "Solving large problem instances of the RWA problem using genetic algorithms". Industrial and Information Systems (ICIIS). Mangalore, Karnataka, India. Vol. 1, pp. 41-46. 2010.

[15] H. Zang, J. Jue, L. Sahasrabuddhe, R. Ramamurthy and B. Mukherjee. "Dynamic lightpath establishment in wavelength routed WDM networks". Communications Magazine IEEE. Vol. 39 Nº 9, pp. 100-108. 2001.

[16] K. Assis, S. Ferreira dos Santos and W. Giozza. "Hybrid Algorithms for Routing and Assignment Wavelengths in Optical Networks". Latin America Transactions, IEEE (Revista IEEE America Latina). Vol. 8 No 3, pp. 214-220. 2010. 